\title{
How competitive intransitivity and niche overlap affect spatial coexistence
}

\author{
Yinghui Yang ${ }^{1}$ and Cang Hui ${ }^{2,3,4}$
}

${ }^{1}$ School of Mathematics, Southwest Jiaotong University, Chengdu 611756, China

${ }^{2}$ Centre for Invasion Biology, Department of Mathematical Sciences, Stellenbosch University, Matieland 7602, South Africa

${ }^{3}$ Biodiversity Informatics Unit, African Institute for Mathematical Sciences, Cape Town

7945, South Africa

${ }^{4}$ International Initiative for Theoretical Ecology, Unit 10, 317 Essex Road, London, N1 2EE, United Kingdom

Corresponding author: Yinghui Yang, School of Mathematics, Southwest Jiaotong University, Chengdu 611756, China. E-mail: yangyh8605@swjtu.edu.cn

\section{Decision date: 03-Nov-2020}

This article has been accepted for publication and undergone full peer review but has not been through the copyediting, typesetting, pagination and proofreading process, which may lead to differences between this version and the Version of Record. Please cite this article as doi: [10.1111/oik.07735]. 


\section{Abstract}

Competitive intransitivity is mostly considered outside the main body of coexistence theories that rely primarily on the role of niche overlap and differentiation. How the interplay of competitive intransitivity and niche overlap jointly affects species coexistence has received little attention. Here, we consider a rock-paper-scissors competition system where interactions between species can represent the full spectra of transitiveintransitive continuum and niche overlap/differentiation under different levels of competition asymmetry. By comparing results from pair approximation that only considers interference competition between neighbouring cells in spatial lattices, with those under the mean-field assumption, we show that (1) species coexistence under transitive competition is only possible at high niche differentiation; (2) in communities with partial or pure intransitive interactions, high levels of niche overlap are not necessary to beget species extinction; and (3) strong spatial clustering can widen the condition for intransitive loops to facilitate species coexistence. The two mechanisms, competitive intransitivity and niche differentiation, can support species persistence and coexistence, either separately or in combination. Finally, the contribution of intransitive loops to species coexistence can be enhanced by strong local spatial correlations, modulated and maximised by moderate competition asymmetry. Our study, therefore, provides a bridge to link intransitive competition to other generic ecological theories of species coexistence.

Keywords: intransitive loop; spatial ecology; species coexistence; niche differentiation; competition asymmetry; pair approximation; mean-field assumption. 


\section{Introduction}

The coexistence and exclusion of co-occurring interacting species has long been a major topic in community ecology (Hutchinson 1961, Tilman 1982). Many theories have been developed based on mechanisms operating between pairs of competitors, such as niche differentiation (MacArthur and Levins 1967, Angert et al. 2009, Chu and Adler 2015). In this regard, the modern coexistence theory (sensu Chesson 2000, Adler et al. 2007) has attributed species coexistence in a community to the balance of niche overlap and competition asymmetry. However, more complex networks of interactions, such as intraguild competition or intransitive competition, are not rare in natural communities. For instance, in a three-species system, an intransitive loop such as a rock-paper-scissors game can form if species A outcompetes $B$, species B outcompetes $C$, and species $C$ outcompetes A. Although a winner can be identified between two species, no definite winner exists in such an intransitive loop, consequently reducing the chance of species extinctions.

The role of intransitive competition in promoting species persistence and coexistence has been studied by many (May and Leonard 1975, Karlson and Jackson 1981, Huisman and Weissing 2001, Reichenbach et al. 2007, Allesina and Levine 2011). The number of involved species in an intransitive loop (i.e. the loop length) was highlighted to play a significant role in regulating species coexistence (Hui et al. 2004, Allesina and Levine 2011, Gallien 2017a). Intransitive networks with an odd number of species often stabilise coexistence by weakening negative density dependence while those formed a loop of even numbers can amplify perturbation, destabilising ecological networks and leading to local extinctions (Allesina and Levine 2011, Vandermeer 2011). Intransitive competition has been observed in many natural systems (Kerr et al. 2002, Huisman and Weissing 1999, Sinervo and Lively 1996, Ulrich et al. 2016), although empirical support for intransitivitymediated coexistence is sparse. Kerr et al. (2002), in particular, tested this coexistence mechanism among microbial laboratory communities by rearranging bacterial cultures within a Petri dish. They found that the diversity of cyclically interacting bacteria could only be maintained with local interactions (i.e. without rearranging bacteria), while the diversity was rapidly lost when interactions happened over relatively large spatial scales (i.e. with rearranging bacteria). Apart from such laboratory experiments (Jackson and Buss 1975, Sinervo and Lively 1996, Lankau and Strauss 2007), the effects of intransitivity on species coexistence have also been inferred from species co-occurrence patterns that conform to these expected from a rock-paper-scissors game (Keddy and Shipley 1989; Freckleton et al. 2000; Ulrich et al. 2014; Soliveres et al. 2015). Since intransitive competition was normally the only contending mechanism in such studies, contrasting results have emerged, fuelling a sustained debate on the role of intransitivity in facilitating species coexistence. The relative contribution of such non-hierarchical interaction loops to biodiversity structuring in communities remains elusive (Grace et al. 1993, Saavedra et al. 2017, Soliveres and Allan 2018).

The integration of intransitive competition into the general species coexistence theory, like niche difference, has been advocated by many (Laird and Schamp 2006, Gallien 2017a, Godoy et al. 2017, Levine et al. 2017, Soliveres and Allan 2018). Several recent studies have made the attempts exactly along this line (Gallien et al. 2017b; Godoy

'This article is protected by copyright. All rights reserved.' 
et al. 2017; Gallien et al. 2018). In particular, Gallien et al. (2017b) built on the classic Lotka-Volterra competition model and developed a metric of the change in invasion growth rate to quantify the contribution of competitive intransitivity to species coexistence. With this metric, they considered an intransitive loop with a fixed level of pairwise niche difference and found that species coexistence can be either stabilised or destabilised depending on the length and the topology of interaction loops. Godoy et al. (2017) then compared two scenarios (with versus without niche difference) of a parameterised model and argued that competitive intransitivity alone does not promote stable coexistence. Weak competitive intransitivity, however, could emerge through adaptive diversification, and intransitivity was found to correlate with trait divergence (thus niche difference) (Gallien et al. 2018). These are studies only starting to build connections between intransitive interactions and traditional coexistence mechanisms. How the contribution of niche difference to intransitivity-mediated coexistence varies across the full spectrum of niche overlap/differentiation remains unknown (Soliveres and Allan 2018).

Spatial structure can profoundly affect ecological and evolutionary systems, modifying species persistence, regulating interaction strengths and species coexistence (Nowak and May 1992, Savill and Hogeweg 1999, Law and Dieckmann 2000, Hui and Li 2004, Yang et al. 2019). However, for the sake of simplicity and tractability, analytical results on competitive intransitivity have generally been developed under well-mixed assumption (Schreiber and Killingback 2013, Gallien et al. 2017b, Godoy et al. 2017, Gallien et al. 2018), often resulting in weak competitive intransitivity (Reichenbach et al. 2007, Laird and Schamp 2015). Intransitivity is indeed less prevalent among mobile taxa in well-mixed environments (Soliveres et al. 2018) such as aquatic protists (Vandermeer 1969), necrophagous insects (Ulrich et al. 2014) and microcosm bacteria (Friedman et al. 2017). In contrast, an important feature of including spatial structure lies in the emergence of self-organised patterns of species distributions which usually act to enhance system stability and species coexistence (Durrett and Levin 1994, May 1994, Kaitala et al. 2001, Johnson and Boerlijst 2002). Laird and Schamp (2008) have demonstrated the case with proliferated diversity when intransitivity-mediated coexistence occurs at local spatial scales. With dispersal limitation, the self-organised mobile clusters and leverage positions in spatial landscapes can explicitly interfere with biotic interactions between species, and the effects of such spatial structure should be considered when formulating intransitivitymediated coexistence (Karlson and Jackson 1981, Johnson and Boerlijst 2002, Zhang et al. 2006, Laird and Schamp 2008; Rojas-Echenique and Allesina 2011, Schreiber and Killingback 2013, Postlethwaite and Rucklidge 2017).

Here, we focus on a three-species interaction loop and extend the classic LotkaVolterra competition model, using pair approximation (PA) and cellular automata, to accommodate local spatial interactions. We then compare results with outcomes from a similar but non-spatial model (the mean-field version of the PA system). Pair approximation has been widely used in theoretical ecology (Matsuda et al. 1992, Webb et al. 2007, Liao et al. 2016, Yang et al. 2019) and is especially powerful to capture dynamics from local interactions between neighbouring individuals (Harada and Iwasa 1994, Hiebeler 2000). It also serves as an approximate analytical tool for spatial lattice models

'This article is protected by copyright. All rights reserved.' 
such as cellular automata (Dieckmann et al. 2000, Webb et al. 2007). Furthermore, as the community matrix of pairwise interactions between all possible pairs of species largely determines the outcome of competition and the chance of species extinction (Hofbauer and Sigmund 1998, Laird and Schamp 2006, Schreiber and Killingback 2013), any changes of the matrix can potentially trigger shifts in the dynamical regimes of the community, switching among oscillation, chaos, stable coexistence and local extinction (Huisman and Weissing 2001). We, therefore, construct a community matrix governed by two parameters. The change of one parameter allows the system to continuously vary from an intransitive loop to a transitive network, while the change of the other parameter determines niche overlap between species. By calculating the change in invasion growth rate, a metric proposed by Gallien et al. (2017b) for quantifying the effect of competitive intransitivity on species coexistence, and evaluating its sensitivity to variation in intransitivity, niche overlap and competition asymmetry, as well as comparing outcomes from pair approximation (representing local spatial interactions) with those from the mean-field assumption, we are able to explore: (1) how niche difference between species affects the contribution of competitive intransitivity to species coexistence; and (2) how competition asymmetry and the spatial structure of species (co-)distributions affect the interplay of competitive intransitivity and niche differentiation, potentially facilitating species coexistence. This, we hope, will establish a theoretical link from competitive intransitivity to existing theories of coexistence that are predominantly based on niche differences and competition asymmetry.

\section{Models}

\section{Pair approximation}

We explore a three-species competition system on a spatial lattice with homogenous habitat. Each site can be either empty ( 0 ) or occupied by one individual of the three species, labelled by 1, 2 and 3 respectively. We assume that each individual can interact with, and send propagules to, $z$ nearest neighbours. Specifically, we considered the von Neumann neighbourhood $(z=4)$. Let $p_{i}$ be the probability (called the global density) of a randomly selected site in state $i(=0,1,2,3)$. Let $p_{i j}$ be the probability (called the doublet density) of a pair of two neighbouring sites being in states $i$ and $j$. Since doublets are not ordered $p_{i j}=p_{j i}$. Let $q_{i / j}$ be the conditional probability (called the local density) of a randomly selected site, neighbouring a site in state $j$, is found in state $i$. By definition, $q_{i / j}=p_{i j} / p_{j}$. Similarly, let $q_{l / i j}$ be the conditional probability of a randomly selected site, neighbouring the site in state $i$ of a doublet of sites in states $i$ and $j$, is found in state $l$, and we have $q_{l / i j}=p_{l i j} / p_{i j}$ ( $p_{l i j}$ represents the corresponding triplet desnity). By definition, we have $\sum_{i \in\{0,1,2,3\}} p_{i}=1, \sum_{j \in\{0,1,2,3\}} p_{i j}=p_{i}$ and $\sum_{j \in\{0,1,2,3\}} q_{j / i}=1$, and consequently we could reduce these probabilities to only nine independent ones: $p_{i}, p_{i i}$ and $p_{i j}$, for $i, j=1,2,3$ and $i<j$. For a large system where these stochastic transition events converge to the continuous rates of their averages (Turchin 2015), the system dynamics are described by the following equations:

$\frac{d p_{i}}{d t}=r_{i}\left(1-\sum_{l=1}^{S} q_{l / i}\right) p_{i}-\left(m_{i}+\sum_{l=1}^{S} \alpha_{l i} q_{l / i}\right) p_{i}$,

'This article is protected by copyright. All rights reserved.' 
$\frac{d p_{i i}}{d t}=2 r_{i} p_{i 0}\left[\lambda+(1-\lambda) q_{i / i 0}\right]-2 p_{i i}\left[m_{i}+\lambda \alpha_{i i}+(1-\lambda) \sum_{l=1}^{s} \alpha_{l i} q_{l / i i}\right]$

$\frac{d p_{i j}}{d t}=(1-\lambda)\left(p_{i 0} r_{j} q_{j / i 0}+p_{j 0} r_{i} q_{i / j 0}\right)-p_{i j}\left[m_{i}+\lambda \alpha_{j i}+(1-\lambda) \sum_{l=1}^{s} \alpha_{l i} q_{l / i j}\right]-p_{i j}\left[m_{j}+\right.$ $\left.\lambda \alpha_{i j}+(1-\lambda) \sum_{l=1}^{S} \alpha_{l j} q_{l / j i}\right]$,

where $r_{i}$ and $m_{i}$ are the intrinsic colonisation rate and death rate of species $i, \alpha_{i j}$ the competition strength of species $i$ on $j, \lambda=1 / z$ the chance of choosing a specific neighbouring lattice, $s=3$ the total number of species. The plus items of equations (1)(3) represent the colonisation of adjacent empty sites, while the minus items stand for mortality from three sources: natural mortality, intra- and inter-specific competition (see Supplementary Material Appendix S1 and Fig.1 for details).

As local density $q_{0 / i}$ characterises the probability of vacant sites surrounding species $i$, while $q_{i / i}$ and $q_{j / i}$ respectively stands for the intra- and interspecific contact probabilities, the ratio $q_{i / i} /\left(q_{j / i}+q_{l / i}\right)(i, j, l=1,2,3$ and $i \neq j \neq l)$ therefore, quantifies the chance of an individual of species $i$ encountering a conspecific individual relative to interacting with an individual of other species $(j$ and $l$ ). If the mean of this ratio over all species is greater than one, the species will have a greater chance to experience local intraspecific competition; otherwise, more interspecific competition is expected.

As competition is assumed to occur only between adjacent individuals, such local interactions are more suitable for modelling sedentary organisms such as terrestrial plants and territorial animals (Gordon 1997, Dieckmann et al. 2000). With this assumption of local interactions between individuals from neighbouring sites, we followed the decoupling moment closure method of pair approximation (Sato et al. 1994, Dieckmann et al. 2000, Webb et al. 2007). According to the pair approximation of local interactions, we set $q_{l / i j} \approx q_{l / i}$ in the above equations so that the dynamical system can be fully expressed by its state variables: $p_{i}, q_{i / i}$ and $q_{i / j}(i<j$ and $i, j=1,2,3$; see

Supplementary Material Appendix S1 for details). The analysis of coexistence conditions is conducted for the above system under the pair approximation.

Please note that the pair approximation does not imply that non-adjacent individuals do not affect each other competitively. Rather, the effect of their interactions can be felt through a cascade of direct interactions between adjacent individuals, often leading to an exponential distance decay of interaction impacts between individuals. Interaction impacts with distance decay much slower (e.g. a power law in mobile species) need to consider higher orders in moment closure.

\section{Competition matrix}

Elements of the competition matrix, $\alpha_{i j}$, describe the impact of competition from species $i$ on $j$. Different competition matrices correspond to different types of competition networks. To explore the role of intransitivity, we set $\alpha_{12}-\alpha_{21}=\alpha_{23}-\alpha_{32}=1-\theta$ (indicating the competition asymmetry between species 1 and 2, and between species 2 and 3, with $\theta=1$ represents symmetric competition among these species) while assigning $\alpha_{31}-\alpha_{13}=1-\theta_{3}$ (Fig.2). If $1-\theta_{3}>0$, species 3 has a competitive advantage over species 1 . Otherwise, species 1 has a competitive advantage over species 3 .

'This article is protected by copyright. All rights reserved.' 
To ensure that the system can continuously change from a pure intransitive loop to a transitive network, we fixed $1-\theta$ to be positive, with $1-\theta_{3}$ ranging from $1-\theta$ (a pure intransitive loop) to $-(1-\theta) / 2$ (akin to intraguild competition), i.e. $\theta_{3}$ ranges within the interval $[\theta, 3 / 2-\theta / 2]$. Parameter $\theta_{3}$, therefore, controls the degree of competitive transitivity (i.e. $1-\theta_{3}$ represents competitive intransitivity), while parameter $\theta$ represents the degree of competition symmetry (i.e. $1-\theta$ represents competition asymmetry).

The degree of niche overlap between competing species $i$ and $j$ can be expressed by a function of intra- and interspecific competition coefficients as $\sqrt{\left(\alpha_{i j} \alpha_{j i}\right) /\left(\alpha_{i i} \alpha_{j j}\right)}$ (Chesson 2000). To ensure the niche overlap between any pair of species $\sqrt{\left(\alpha_{i j} \alpha_{j i}\right) /\left(\alpha_{i i} \alpha_{j j}\right)} \equiv k$, we assigned the intraspecific competition coefficients as $\alpha_{11}=\sqrt{\theta_{3}} / k, \alpha_{22}=\theta /\left(k \sqrt{\theta_{3}}\right)$, and $\alpha_{33}=\sqrt{\theta_{3}} / k$. In particular, we chose the degree of niche overlap $k$ from $[0,0.9]$. We therefore have the following competition matrix for the three-species network:

$M=\left(\begin{array}{ccc}\frac{\sqrt{\theta_{3}}}{k} & 1 & \theta_{3} \\ \theta & \frac{\theta}{k \sqrt{\theta_{3}}} & 1 \\ 1 & \theta & \frac{\sqrt{\theta_{3}}}{k}\end{array}\right)$.

Evidently, how the three species perform and coexist depend on three parameters: niche overlap $k$, competitive intransitivity $1-\theta_{3}$ and competition asymmetry $1-\theta$. Note, to highlight the roles of these three parameters, we set the intrinsic colonisation rate $r_{i}$ and death rate $m_{i}$ to be equal among species.

\section{Change in invasion growth rate}

To assess the impact of intransitivity on the outcome of competition, we computed the average change in invasion growth rate, $\overline{\Delta R}$, proposed by Gallien et al. (2017b). The change in invasion growth rate for species $i$ is defined as the following:

$\Delta R_{i}=\frac{\sum_{j \neq i, j=1}^{S}\left(R_{i}-R_{i,-j}\right)}{s-1}$,

where $R_{i}$ is the invasion growth rate of species $i$ when other species are set at their equilibrium while species $i$ is invading the system with an extremely small propagule size; $R_{i,-j}$ represents the invasion growth rate of species $i$ in the same system after removing species $j$. The term $R_{i}-R_{i,-j}$ suggests the invasion growth rate change for species $i$ caused by the elimination of species $j$. As such, $\Delta R_{i}$ describes the average change in the invasion growth rate of species $i$ due to the removal of any other species in the system. If the system is an intransitive loop, the removal of one resident species can break the loop of interactions and reduce the overall invasion growth rate, while the opposite is true if species compete hierarchically with nonzero niche overlaps (Gallien et al. 2017b). For species 1 , for instance, we first calculated $R_{1,-3}$ (removing species 3 ), 
$R_{1,-2}$ (removing species 2), $R_{1}$ (without removing other species) and then $\Delta R_{1}$ according to the above formula. In our case, the invasion growth rates $R_{i}$ was derived as the per-capita population growth rate $\left(d p_{i} / d t\right) / p_{i}$ when its population size was infinitesimal and the population sizes of other species at their equilibrium. The average change in the invasion growth rate of all species was finally computed, $\overline{\Delta R}=\left(\Delta R_{1}+\right.$ $\left.\Delta R_{2}+\Delta R_{3}\right) / 3$; see detail in Supplementary Material Appendix S2. Consequently, $\overline{\Delta R}>0$ indicates the positive contribution of intransitivity to species coexistence, while $\overline{\Delta R}<0$ reflects the negative contribution of transitive interactions to species coexistence.

\section{Mean field assumption}

Mean field assumption is normally implemented when modelling the dynamics of a wellmixed system or one that does not exclusively reflect local interactions (Zhang et al. 2006). For comparison, we implemented the mean field assumption in our system by equating local densities to their corresponding global densities, $q_{l / i}=p_{i l} / p_{i} \approx p_{l}(l=1,2,3)$. This simplifies the model to the following,

$\frac{d p_{i}}{d t}=r_{i}\left(1-\sum_{l=1}^{S} p_{l}\right) p_{i}-\left(m_{i}+\sum_{l=1}^{s} \alpha_{l i} p_{l}\right) p_{i}$.

This is similar to the model proposed by Taneyhill (2000). To compare with results from pair approximation, we also explored the effects of competitive transitivity $\left(\theta_{3}\right)$ and niche overlap $(k)$ in this mean-field system on the contribution of competitive intransitivity to species coexistence $(\overline{\Delta R})$ (see Supplementary Material Appendix S3).

\section{Cellular automata}

To verify the emergence of spatial patterns in our pair-approximation system, we developed cellular automata over a regular $n \times n$ square lattices $(n=100)$ with periodic boundary conditions. As the implementation of cellular automata inevitably introduces additional stochasticity into the system, we chose the large number of lattices and the periodic boundary to reduce the stochasticity and any boundary effects. Consequently, the stochastic dynamics from the cellular automata should converge to the rate dynamics depicted by the pair approximation. At each time step, the states of all lattice cells $(=0,1,2,3$, representing empty and occupied cells by corresponding species) were updated simultaneously. Individuals in each cell can only interact with their nearest four neighbours (von Neumann neighbourhood), similar to the case of pair approximation. As the initial condition of lattice states has a negligible effect on the model output (Hiebeler and Morin 2007), we randomly assigned the initial states to lattice cells with equal chance. The specific transition rules of cell states between consecutive time steps are provided in Table 1. The cellular automata were run until all global densities have reached equilibrium. We used the method in Yang et al. (2014) to evaluate whether the system has reached equilibrium by monitoring and comparing global densities every 100 time steps after an initial run of 1000 time steps. The system is considered to have reached its equilibrium if the differences in global densities between these runs with gaps of 100 time steps become less than 0.1 (Hiebeler et al. 2016). 


\section{Results}

\section{Coexistence mediated by competitive intransitivity and niche differentiation}

We continuously shifted the system from a transitive network $\left(\theta_{3}=(3-\theta) / 2\right)$ to a pure intransitive competition loop $\left(\theta_{3}=\theta\right)$ (Fig.2). The average change in invasion growth rate $\overline{\Delta R}$ was calculated along two spectra: competitive transitivity $\left(\theta_{3}\right)$ and niche overlap $(k)$. As shown in Fig.3a, there exists a critical transition in the parameter space (around $\theta_{3}=0.4+0.6 k$, from interpolation), where the contribution of competitive intransitivity to species coexistence shifts from positive $(\overline{\Delta R}>0$, area with reddish colours where $\left.\theta_{3}<0.4+0.6 k\right)$ to negative $\left(\overline{\Delta R}<0\right.$, area with blueish colours where $\left.\theta_{3}>0.4+0.6 k\right)$. Therefore, competitive intransitivity only facilitates species coexistence when both the levels of intransitivity and niche overlap are high (in other words, a high intransitivity and low niche differentiation).

Fig. $3 \mathrm{~b}$ shows the bifurcation condition between species coexistence and extinction. We noted that the range of transitivity $\left(\theta_{3}\right)$ to trigger species extinction has widened (i.e. the range of intransitivity to facilitate species coexistence has narrowed) with the increase of niche overlap from moderate to high levels. However, increasing niche overlap $(k)$ does not necessarily lead to species extinction when the system harbours high or low intransitivity (small $\theta_{3}$ ). In particular, when the system is transitive $\left(\theta_{3}>1\right.$; akin to intraguild competition), competition favours species 1 over 3 . High niche overlap would impose a high level of competition pressure to species 3. In such a transitive network, stable coexistence is only possible under low levels of niche overlap. In other words, niche differentiation is necessary for species to stably coexist in transitive networks. In contrast, in an intransitive community $\left(\theta_{3}<1\right)$, species coexistence can be achieved under the full spectrum of niche overlap.

Three scenarios of species coexistence can be clarified in the parameter space of Fig.3 from the interplay of two mechanisms - competitive intransitivity and niche differentiation. First (left hand of the plot), with a high niche differentiation $(k \rightarrow 0)$, intraspecific competition becomes much greater than interspecific competition, supporting the traditional coexistence theory of niche differentiation. Second (right hand of the plot), with a high niche overlap (e.g. $k=0.9$ ) that has reduced the competition asymmetry (see next section), some level of intransitivity is still needed to ensure species coexistence. Finally (rest of the plot), niche differentiation and competitive intransitivity can supplement each other and jointly promote species coexistence under moderate niche overlap $(0.3<k<0.8)$.

\section{Competition asymmetry and local interactions}

To explore the robustness of above results under different levels of competition asymmetry between species that is proportional to $1-\theta$, we repeated the above analyses of our system under pair approximation at different levels of $\theta$, ranging from 0.3 to 0.7 (Fig.4a). We compared the results with corresponding ones from the system under the mean-field assumption (Fig.4b).

'This article is protected by copyright. All rights reserved.' 
It is evident that species coexistence is not possible in systems lacking intransitivity but with high niche overlap (top right corner of the plot), and the parameter zone for extinction steadily enlarges when moving from a neutral community (no competition asymmetry between species with $\theta$ close to 1 ) to a community dominated by asymmetric competition ( $\theta$ close to 0 ). Competition asymmetry was also found to strongly affect how much intransitivity promotes species coexistence. Highly symmetric competition (i.e. neutral competition with $\theta$ close to 1 in Fig.4a) resulted in narrowing the parameter range of intransitivity-facilitated coexistence (reddish part in the bottom right of the plot). However, excessive competition asymmetry can also hamper intransitivity-facilitated coexistence which is maximised with a moderate competition asymmetry in communities of local interactions $(\theta=0.5$ in Fig.4a). In general, compared to well-mixed interactions, local interactions allow wider parameter ranges for competitive intransitivity to facilitate species coexistence. In particular, for a low to medium level of niche overlap ( $0 \leq k \leq 1$ / 2), a range matching variation of niche overlap in natural systems (Chu and Adler 2015), we found little intransitivity-mediated coexistence in well-mixed systems. More details on how intransitive loops affect the outcome of competition between species under the mean-field assumption for well-mixed interactions are provided in Supplementary Material Appendix S3 and Fig. S1.

\section{Emergence of spatial patterns}

From the systems with local interactions under pair approximation, we explored the spatial patterns by first using equilibria of local density $q_{0 / i}^{*}$ depicting the degree to which species $i$ is surrounded by empty cells, and the ratio of intraspecific to interspecific interaction rates, $q_{i / i}^{*} /\left(q_{j / i}^{*}+q_{l / i}^{*}\right)$ (Fig.5). In Fig.5, we see that with the increase of niche overlap, both the ratios of intra- to interspecific interaction rates (Fig.5a) and the local densities adjacent to empty cells (Fig.5b) have increased. This suggests that with the increase of niche overlap and the intensified interspecific competition as a consequence, species become increasingly isolated from each other in space, forming a pattern of being globally rare but locally abundant. As the intensity of intransitive competition depends on local densities, rare species can still garner the benefit of intransitivity from being locally abundant. However, with well-mixed interactions under the mean-field assumption, interference competition (i.e. $\alpha_{l i} p_{l} p_{i}$ in equation (6)) depends purely on global densities $\left(p_{l}\right.$ and $\left.p_{i}\right)$, making a rare species with a low global density difficult to establish an effective intransitive loop and to rebound. As such, it is rather difficult for competitive intransitivity to facilitate species coexistence in communities with well-mixed interactions.

With local interactions, the narrowing parameter region of intransitivity-mediated coexistence in Fig.4 under increasing levels of competition asymmetry (smaller $\theta$ values) can be explained by those curves in Fig.5. Notably, communities with higher competition asymmetry are more sensitive to the increase of niche overlap (blue curve above red one in Fig.5 for low to moderate levels of niche overlap). This is because, with the increase of niche overlap, the intensity of interspecific competition increases in communities experiencing greater competition asymmetry, which then results in more empty cells and

'This article is protected by copyright. All rights reserved.' 
segregated species distributions. However, further increases in niche overlap after its level has already reached a moderately high level $(k>0.5$; Fig.5) can flatten or even reduce the probabilities of an individual surrounded by empty cells or individuals from other species (Fig.5). This is largely due to the formation of segregated species distributions that hamper the establishment of local intransitive loops. Without the intransitive loops facilitating species coexistence, population sizes are expected to drop, further reducing the local encounter rate between individuals of different species.

Above analytic results for communities with local interactions have been validated by simulations from cellular automata (Fig.6). In particular, with moderate competition asymmetry $(\theta=0.6)$ and purely intransitive interactions $\left(\theta_{3}=\theta\right)$, we explored the spatial dynamics under three levels of niche overlap: $k=0.01$ (low), 0.25 (medium) and 0.8 (high). As shown in Fig.6, both niche differentiation and competitive intransitivity can facilitate species coexistence, but in different ways. With little niche overlap ( $k=0.01$ ), intraspecific interactions become the dominant force that can drive species distributions to become scattered and well mixed. In this scenario species coexistence is predominantly maintained by niche differentiation (left column in Fig.6). In contrast, with medium or high levels of niche overlap ( $k=0.25,0.8$; middle and right columns in Fig.6), the spatial dynamics are not static but constantly evolving. Strong interspecific competition creates both a certain number of empty sites and more clustered distributions of species. In such case, species coexistence is largely maintained by local cyclic interactions. Finally, compared with systems with lower levels of niche overlap, species with smaller equilibrium population sizes in systems with high niche overlap also often experience much greater fluctuations (bottom row in Fig.6). This is because pure intransitivity with large competition asymmetry can typically produce population oscillation (Vandermeer 2011), while a moderate level of niche difference, even if not strong enough to stabilise coexistence, can still slow down competitive elimination (Gallien et al. 2017b) by suppressing population oscillation.

\section{Discussion}

\section{Interplay of intransitivity and niche differentiation}

We have developed here a three-species competition model with local interactions using pair approximation and investigated the interplay of niche differentiation and competitive intransitivity in facilitating species coexistence. By formulating local densities that describe the rates of intra- and interspecific interactions, we have effectively captured the community dynamics under local interactions. Unlike systems with well-mixed interactions that were formulated under the mean-field assumption and suggested little role of competitive intransitivity at play in facilitating species coexistence besides niche differentiation (Gallien et al. 2017b, Godoy et al. 2017), our model suggests that both mechanisms are important in communities with local interactions. The specific way that these two mechanisms interplay in supporting species coexistence depends on how the levels of niche overlap and competition asymmetry play out in local interactions.

Transitive communities cannot ensure species coexistence in the absence of niche difference. Communities with stronger transitivity demand a higher degree of niche 
difference to allow species coexistence. Consequently, species extinctions tend to occur in communities with a high niche overlap and strong transitive interactions (Fig.3). In such cases, the condition for species coexistence is akin to those of a three-species module engaging in intraguild predation or competition, with one intraguild predator, intraguild prey and a shared resource species. Standard models on intraguild predation suggest that species are less likely to stably coexist with each other unless the intraguild prey can consume the shared resource more efficiently than the intraguild predator (Holt and Polis 1997). A subsequent work from Holt and Huxel (2007) further proved that exclusive resources that can only be accessed by the intraguild prey or predator can alter this restriction on species coexistence.

For systems with competitive intransitivity, we have shown that strong niche overlap does not necessarily lead to species extinction. Rather, species coexistence can be modulated by pairwise niche difference, competitive intransitivity, or both. Specifically, with little niche overlap, intraspecific conflicts are more important than interspecific interactions in regulating community assembly, as highlighted in the 'modern coexistence theory' (Chesson 2000, Chesson and Kuang 2008). Such an overemphasis on intraspecific interaction, inevitably, downplays the role of interaction structure, be it transitive or intransitive, in regulating species coexistence and community stability. In such cases, niche difference becomes the sole mechanism that explains species coexistence.

With high levels of niche overlap when species are competing for similar resources, competitive intransitivity entangles species to become 'ecologically equivalent' and consequently mitigates the chance of extinction from interspecific competition (Laird and Schamp 2006), much like the assumption of ecologically equivalent species in the neutral model (Hubbell 2001). Although neutral dynamics can explain a number of community assembly patterns (Chave et al. 2002, Tilman 2004, Purves and Pacala 2005), its assumption of ecological equivalence between species might not be common in nature (Holt 2006, McGill et al. 2006). Competition, and more generally the network of biotic interactions, plays an important role in explaining species distributions and community assembly patterns (Grime 1979, Schoener 1983, Gotelli and McCabe 2002, Bosc et al. 2018, Latombe et al. 2018, Hui and Richardson 2019, Steidinger et al. 2019). As competitive intransitivity can allow species to coexist without neglecting the importance of competition itself in community assembly (Soliveres et al. 2018), it is time to expand the coexistence theory in network and community ecology to incorporate competitive intransitivity (Laird and Schamp 2006).

With partial niche difference between species, both mechanisms (niche differentiation and competitive intransitivity) can jointly promote species coexistence. Although weak intransitivity alone is not sufficient to counteract the pressure from competitive exclusion, niche differentiation can compensate the need for stabilising force to allow species coexistence (as shown in Fig.3). This suggests that the evidence of intransitive interactions found in many species-rich communities does not mean it the sole mechanism of diversity maintenance, especially when species niches differ. Downplaying niche-related mechanisms can overstate the contribution of competitive intransitivity to species coexistence. To date, competitive intransitivity has been documented in many ecological systems (Keddy and Shipley 1989, Laird and Schamp 2006, Godoy et al. 2017, Soliveres

'This article is protected by copyright. All rights reserved.' 
and Allan 2018), while our results call for more attention on the condition for intransitivity to work effectively in promoting species coexistence.

\section{Competition asymmetry in local interactions}

Competition asymmetry between species can greatly modulate the contribution of competitive intransitivity to species coexistence in communities with local interactions. Along with the typical 'equalising mechanism' (Chesson 2000, Tilman 2004), suppressing interspecific competition relative to intraspecific competition enables coexistence (e.g. Barot and Gignoux 2004). However, in such a parameter range, our results suggest that the contribution of competitive intransitivity is rather weak $(\theta=0.7$ in Fig.4a), with positive influence only detected in a pure intransitive loop, although such a weak contribution from intransitivity could also be due to the lack of notable competition asymmetry. As symmetric competition leads to fitness equivalence, the destabilising force of transitive interactions is also minimised. In such a case, population dynamics is largely neutral and controlled by drift and stochasticity due to equivalent fitness and similar niches between species (Adler et al. 2007). As competition asymmetry increases, the contribution of competitive intransitivity starts to rise $(\theta=0.5 \sim 0.6$ in Fig.4a). Although we expected increased contribution from competitive intransitivity with widening competition asymmetry (according to the mean-field model), our pair-approximation model depicting local interactions suggested that this is not always the case. Highly asymmetric competition can eventually reduce the intransitivity-mediated coexistence $(\theta=0.3$ in Fig.4a).

The facilitating role of competitive intransitivity was maximised only with a moderate level of competition asymmetry, contrasting with mean-field results. This can be explained by the high conspecific aggregation resulting from excessive interspecific competition due to large competition asymmetry. Without empty sites or local spaces to afford multiple species adjacent to each other, the 'rock-paper-scissors' interaction loop cannot be assembled locally. If only two of the three species can simultaneously establish at a local area but without involving the third species, one of the established two can be competitively excluded (Gallien et al. 2017b). In such a scenario of local interactions, cyclic competition can only contribute to species coexistence within a narrow parameter range. Nevertheless, the parameter range for intransitivity-mediated coexistence has been widened under local interactions (pair approximation) when compared to the one under well-mixed interactions (mean-field assumption). As such, we attribute the enhanced intransitivity-mediated coexistence to local interactions.

Dispersal limitation and spatial neighbourhood structure can have profound effects on population and community dynamics, especially for sedentary species such as plants that competing for resources at local scales (Harper 1977, Boots and Sasaki 2000, Dieckmann et al. 2000, Liao et al. 2013). Studies under the mean-field assumption usually assume competition intensity between species proportional to the encounter rate (product of the global densities of involved species), reflecting well-mixed interactions (Keddy and Shipley 1989, Ying et al. 2014). This contrasts with the non-random (co)distributions of most species (Condit et al. 2000, Bell 2005, Wang et al. 2010). Instead, our pair-approximation model only describes dispersal and interactions between directly

'This article is protected by copyright. All rights reserved.' 
adjacent cells (Stoll and Weiner 2000), with the death rate reflecting mostly the impact from local competitors rather than the overall effect of global densities (Dieckmann et al. 2000). Due to the fitness difference from niche overlap, conspecific individuals tend to form spatial clumps (Phillips and MacMahon 1981). Therefore, globally rare species can be locally abundant, which allows all species of an intransitive loop to coexist. However, such cyclic competition can trigger large fluctuations of species population density especially for those species with a low density at equilibrium (Fig.6). Indeed, as long as such segregated distributions are maintained, interspecific competition will be suppressed more than intraspecific competition, thus promoting stable coexistence (Murrell and Law 2003, Yang et al. 2014). With such clumped and segregated spatial distributions, some have shown intransitivity-mediated coexistence can be enhanced (Edwards and Schreiber 2010, Ulrich et al. 2017, Yitbarek and Vandermeer 2017). For instance, competitive intransitivity conbined with life-histoy tradeoffs have been documented in spatially structured communities (Edwards and Schreiber 2010). Dispersal limitation alone could suffice to generate spatial aggregation and thus promote species coexistence (Yitbarek and Vandermeer 2017). We here, by contrast, have highlighed the role of local interactions, together with competitive intransitivity and niche difference, in promoting species coexistence in spatial communities.

Biotic interactions in nature are highly complex. We here only modelled pairwise interactions among three species. Higher-order interactions have been shown to greatly affect the stability of species-rich communities (Grilli et al. 2017, Mayfield and Stouffer 2017). While higher-order interactions can promote species coexistence and stabilise randomly assembled communities including those with intransitive loops (Grilli et al. 2017), a generic platform to study higher-order interactions has also been proposed to partition fitness into additive linear components (as in most Lotka-Volterra type models) and higher-order components (including both intraspecific and interspecific higher order interactions) (Mayfield and Stouffer 2017). Research on the role of higher-order interactions is only starting to gain momentum, with a set of metrics that empirical ecologists can use yet to be proposed, a challenge highlighted by Mayfield and Stouffer (2017). How Chesson's (2000) pairwise niche overlap metric can be further developed to measure higher-order interactions remains to be seen. Besides, the greater impact of higher-order interactions to community stability needs to be counterbalanced by the fact that, with the time resolution increasing, the encounter rate of higher-order interactions can become negligible much faster than the encounter rate of pairwise interactions. Moreover, a species-rich community can harbour multiple interaction loops (intransitive or transitive cycles). For instance, a community with three species (as in our model) can at most form one intransitive loop (e.g. $1 \rightarrow 2 \rightarrow 3 \rightarrow 1$ ), while a community with four species can already include, at maximum, three intransitive loops (e.g. $1 \rightarrow 2 \rightarrow 3 \rightarrow 4 \rightarrow 1,1 \rightarrow 2 \rightarrow 3 \rightarrow 1$, $1 \rightarrow 2 \rightarrow 4 \rightarrow 1$ ). This can greatly affect the system stability and therefore species coexistence. Studies on quantitative loop analyses seem to provide a tentative platform (Levins 1966, 1975, Justus 2005). Both higher-order interactions and complex interaction networks need to be considered in future studies to explore the role of interaction intransitivity in steering community assembly and facilitating species coexistence.

'This article is protected by copyright. All rights reserved.' 


\section{Concluding remarks}

Our models have weaved competitive intransitivity into standard coexistence theories. By doing so, we could obtain a deeper mechanistic understanding of species coexistence. The pair approximation allowed us to explore the role of competitive intransitivity and other related coexistence mechanisms (niche differentiation and fitness equivalence) in communities with local interactions. We show that competitive intransitivity can become a dominant mechanism for species coexistence and biodiversity maintenance under high levels of niche overlap. Such an intransitivity-mediated coexistence could be strengthened in communities with moderate competition asymmetry, such as the sagebrush steppe community of Idaho (with a high level of niche overlap, $k=0.41$, and a moderate level of competition asymmetry, 1.59 over the range from 1.02 to 2.54 , which corresponds to $\theta=0.375$ in our scaled range from zero to one; Chu and Adler 2015). In such communities, future studies should thus focus more on the loss of species. Should the lost species be involved in intransitive loops, it might have greatly disrupted the initial intransitivity-mediated coexistence, potentially leading to extinction cascades. Here, we only investigated the simplest three-species system. In a species-rich community, more than one intransitive loop can be established (Buss and Jackson 1979, Soliveres et al. 2015). The length, number and nested structure of loops can all play significant roles in regulating community stability (Huisman and Weissing 2001, Szabo and Czaran 2001, Allesina and Levine 2011). However, methods to accommodate more species for reliable predictions of assemblage-level dynamics is not a simple task (Hui and Richardson 2019). Future work should focus more on unveiling the finer structure of interaction matrices and linking network structure to the interplay of competitive intransitivity and niche difference.

Acknowledgements - We are grateful to Bang-sheng Han, François Massol and three anonymous reviewers for constructive comments.

Funding - YY is supported by the National Natural Science Foundation of China (NSFC grants

31700347 and 11801470). CH is supported by the National Research Foundation of South Africa (89967).

Conflicts of interest - We declare no conflicts of interest.

Author contributions - YY conceived the study, developed the initial models and analyses, $\mathrm{CH}$ provided additional model analyses, and both authors contributed to the writing and editing of the manuscript.

\section{References}

Adler, P. B. et al. 2007. A niche for neutrality. - Ecol. Lett. 10: 95-104. 
Allesina, S. and Levine, J. M. 2011. A competitive network theory of species diversity. - P. Natl. Acad. Sci. USA 108: 5638-5642.

Angert, A. L. et al. 2009. Functional tradeoffs determine species coexistence via the storage effect. - P. Natl. Acad. Sci. USA. 106: 11641-11645.

Barot, S. and Gignoux, J. 2004. Mechanisms promoting plant coexistence: can all the proposed processes be reconciled? - Oikos 106: 185-192.

Bell, G. 2005. The co-distribution of species in relation to the neutral theory of community ecology. - Ecology 86: 1757-1770.

Boots, M. and Sasaki, A. 2000. The evolutionary dynamics of local infection and global reproduction in host-parasite interactions. - Ecol. Lett. 3: 181-185.

Bosc, C. et al. 2018. Interactions among predators and plant specificity protect herbivores from top predators. - Ecology 99: 1602-1609.

Buss, L. W. and Jackson, J. B. C. 1979. Competitive networks: nontransitive competitive relationships in cryptic coral reef environments. - Am. Nat. 113: 223-234.

Chave, J. et al. 2002. Comparing classical community models: Theoretical consequences for patterns of diversity. - Am. Nat. 159: 1-23.

Chesson, P. 2000. Mechanisms of maintenance of species diversity. - Annu. Rev. Ecol. Syst. 31: 343-366.

Chesson, P. and Kuang, J. J. 2008. The interaction between predation and competition. Nature 456: 235-238.

Chu, C. and Adler, P. B. 2015. Large niche differences emerge at the recruitment stage to stabilize grassland coexistence. - Ecol. Monogr. 85: 373-392. 
Condit, R. et al. 2000. Spatial patterns in the distribution of tropical tree species. - Science 288: $1414-1418$.

Dieckmann, U. et al. 2000. The geometry of ecological interactions: simplifying spatial complexity. - Cambridge Univ. Press.

Durrett, R. and Levin, S. 1994. The importance of being discrete (and spatial). - Theor. Popul. Biol. 46: 363-394.

Edwards, K. F. and Schreiber, S. J. 2010. Preemption of space can lead to intransitive coexistence of competitors. - Oikos 119: 1201-1209.

Freckleton, R.P. et al. 2000. Determinants of the abundance of invasive annual weeds: community structure and non-equilibrium dynamics. - Proc. R. Soc. B 267: 1153- 1161

Friedman, J. et al. 2017. Community structure follows simple assembly rules in microbial microcosms. - Nat. Ecol. Evol. 1: 0109

Gallien, L. 2017a. Intransitive competition and its effects on community functional diversity. - Oikos 126: 615-623.

Gallien, L. et al. 2017b. The effects of intransitive competition on coexistence. - Ecol. Lett. 20: 791-800.

Gallien, L. et al. 2018. Emergence of weak-intransitive competition through adaptive diversification and eco-evolutionary feedbacks. - J. Ecol. 106: 877-889.

Godoy, O. et al. 2017. Intransitivity is infrequent and fails to promote annual plant coexistence without pairwise niche differences. - Ecology 98: 1193-1200.

Gordon, D. 1997. The population consequences of territorial behavior. - Trends Ecol. Evol. 12: 63-66. 
Gotelli, N. J. and McCabe, D. J. 2002. Species co-occurrence: A meta-analysis of J. M. Diamond's assembly rules model. - Ecology 83: 2091-2096.

Grace, J. B. et al. 1993. The examination of a competition matrix for transitivity and intransitive loops. - Oikos 68: 91-98.

Grilli, J. et al. 2017. Higher-order interactions stabilize dynamics in competitive network models. - Nature 548:210-213.

Grime, P. 1979. Plant strategies and vegetation processes. - Wiley.

Harada, Y. and Iwasa, Y. 1994. Lattice population dynamics for plants with dispersing seeds and vegetative propagation. - Res. Popul. Ecol. 36: 237-249.

Harper, J. L. 1977. Population biology of plants. - Academic Press.

Hiebeler, D. 2000. Populations on fragmented landscapes with spatially structured heterogeneities: Landscape generation and local dispersal. - Ecology 81: 1629-1641.

Hiebeler, D. E. and Morin, B. R. 2007. The effect of static and dynamic spatially structured disturbances on a locally dispersing population. - J. Theor. Biol. 246: 136-144.

Hiebeler, D. E. et al. 2016. Locally dispersing populations in heterogeneous dynamic landscapes with spatiotemporal correlations. I. Block disturbance. - J. Theor. Biol. 407: $212-224$

Hofbauer, J. and Sigmund, K. 1998. Evolutionary games and population dynamics. Cambridge Univ. Press.

Holt, R. D. 2006. Emergent neutrality. - Trends Ecol. Evol. 21: 531-533.

Holt, R. D. and Polis, G. A. 1997. A theoretical framework for intraguild predation. - Am. Nat. 149: 745-764.

'This article is protected by copyright. All rights reserved.' 
Holt, R. D. and Huxel, G. R. 2007. Alternative prey and the dynamics of intraguild predation: Theoretical perspectives. - Ecology 88: 2706-2712.

Hubbell, S. P. 2001. The unified neutral theory of biodiversity and biogeography. Princeton Univ. Press.

Hui, C. and Li, Z. Z. 2004. Distribution patterns of metapopulation determined by Allee effects. - Popul. Ecol. 46: 55-63.

Hui, C. and Richardson, D. M. 2019. How to invade an ecological network. - Trends Ecol. Evol. 34: 121-131.

Huisman, J. and Weissing, F. J. 1999. Biodiversity of plankton by species oscillations and chaos. - Nature 402: 407-410.

Huisman, J. and Weissing, F. J. 2001. Biological conditions for oscillations and chaos generated by multispecies competition. - Ecology 82: 2682-2695.

Hutchinson, G. E. 1961. The paradox of the plankton. - Am. Nat. 95: 137-145.

Jackson, J. B. C. and Buss, L. 1975. Allelopathy and spatial competition among coral reef invertebrates.-Proc. Nat. Acad. Sci. USA 72: 5160-5163.

Johnson, C. R. and Boerlijst, M. C. 2002. Selection at the level of the community: the importance of spatial structure. - Trends Ecol. Evol. 17: 83-90.

Justus, J. 2005. Qualitative Scientific Modeling and Loop Analysis. -Philos. Sci. 72: 12721286.

Kaitala, V. et al. 2001. Self-organized dynamics in spatially structured populations. - Proc. R. Soc. B. 268: 1655-1660. 
Karlson, R. H. and Jackson, J. B. C. 1981. Competitive networks and community structure: a simulation study. - Ecology 62: 670-678.

Keddy, P. A. and Shipley, B. 1989. Competitive hierarchies in herbaceous plant communities. - Oikos 54: 234-241.

Kerr, B. et al. 2002. Local dispersal promotes biodiversity in a real-life game of rock-paperscissors. - Nature 418: 171-174.

Laird, R. A. and Schamp, B. S. 2006. Competitive intransitivity promotes species coexistence. - Am. Nat. 168: 182-193.

Laird, R. A. and Schamp, B. S. 2008. Does local competition increase the coexistence of species in intransitive networks? - Ecology 89: 237-247.

Laird, R. A. and Schamp, B. S. 2015. Competitive intransitivity, population interaction structure, and strategy coexistence. - J. Theor. Biol. 365: 149-158.

Lankau, R. A., and Strauss, S. Y. 2007. Mutual feedbacks maintain both genetic and species diversity in a plant community. - Science 317:1561-1563.

Latombe, G. et al. 2018. Drivers of species turnover of native and alien plants with different residence times vary with species commonness. - Ecology 99: 2763-2775.

Law, R. and Dieckmann, U. 2000. A dynamical system for neighborhoods in plant communities. - Ecology 81: 2137-2148.

Levine, J. M. et al. 2017. Beyond pairwise mechanisms of species coexistence in complex communities. - Nature 546: 56-64.

Levins, R. 1975. Evolution in communities near equilibrium. - In: Cody, M., Diamond J. (ed.), Ecology and evolution of communities. Belknap, pp. 16-50. 
Levins, R. 1966. The strategy of model building in population biology. - Am. Sci. 54: 421431.

Liao, J. B. et al. 2013. Species persistence in landscapes with spatial variation in habitat quality: A pair approximation model. - J. Theor. Biol. 335: 22-30.

Liao, J. B. et al. 2016. Coexistence of species with different dispersal across landscapes: a critical role of spatial correlation in disturbance. - Proc. R. Soc. B 283: 20160537.

MacArthur, R. and Levins, R. 1967. The limiting similarity, convergence, and divergence of coexisting species. - Am. Nat. 101: 377-385.

Matsuda, H. et al. 1992. Statistical-mechanics of population - the lattice Lotka-Volterra model. - Prog. Theor. Phys. 88: 1035-1049.

May, R. M. 1994. Spatial chaos and its role in ecology and evolution. - In: Levin, S. A. (ed.) Frontiers in mathematical biology. Springer, pp. 89-103.

May, R. M. and Leonard, W. J. 1975. Nonlinear aspects of competition between three species. - SIAM J. Appl. Math. 29: 243-253.

Mayfield, M. M. and Stouffer, D. B. 2017. Higher-order interactions capture unexplained complexity in diverse communities. - Nat. Eco. Evo. 1:0062.

McGill, B. et al. 2006. Empirical evaluation of neutral theory. - Ecology 87: 1411-1423.

Murrell, D. J. and Law, R. 2003. Heteromyopia and the spatial coexistence of similar competitors. - Ecol. Lett. 6: 48-59.

Nowak, M. A. and May, R. M. 1992. Evolutionary Games and Spatial Chaos. - Nature 359: 826-829. 
Phillips, D. L. and MacMahon, J. A. 1981. Competition and Spacing Patterns in Desert Shrubs. - J. Ecol. 69: 97-115.

Postlethwaite, C. M. and Rucklidge, A. M. 2017. Spirals and heteroclinic cycles in a spatially extended Rock-Paper-Scissors model of cyclic dominance. - Europhys Lett. 117: 48006.

Purves, D. W. and Pacala, S. W. 2005. Ecological drift in nichestructured communities: neutral pattern does not imply neutral process. - In: Burslem, D., et al. (eds.), Biological interactions in the tropics. Cambridge University Press, pp. 107-140.

Reichenbach, T. et al. 2007. Mobility promotes and jeopardizes biodiversity in rock-paperscissors games. - Nature 448: 1046-1049.

Rojas-Echenique, J. and Allesina, S. 2011. Interaction rules affect species coexistence in intransitive networks. - Ecology 92: 1174-1180.

Saavedra, S. et al. 2017. A structural approach for understanding multispecies coexistence. - Ecol. Monogr. 87: 470-486.

Sato, K. et al. 1994. Pathogen invasion and host extinction in lattice structured populations. - J. Math. Biol. 32: 251-268.

Savill, N. J. and Hogeweg, P. 1999. Competition and dispersal in predator-prey waves. Theor. Popul. Biol. 56: 243-263.

Schoener, T. W. 1983. Field experiments on interspecific competition. - Am. Nat. 122: 240285.

Schreiber, S. J. and Killingback, T. P. 2013. Spatial heterogeneity promotes coexistence of rock-paper-scissors metacommunities. - Theor. Popul. Biol. 86: 1-11. 
Sinervo, B. and Lively, C. M. 1996. The rock-paper-scissors game and the evolution of alternative male strategies. - Nature 380: 240-243.

Soliveres, S. and Allan, E. 2018. Everything you always wanted to know about intransitive competition but were afraid to ask. - J. Ecol. 106: 807-814.

Soliveres, S. et al. 2015. Intransitive competition is widespread in plant communities and maintains their species richness. - Ecol. Lett. 18: 790-798.

Soliveres, S. et al. 2018. Intransitive competition is common across five major taxonomic groups and is driven by productivity, competitive rank and functional traits. - J. Ecol. 106: 852-864.

Steidinger, B.S. et al. 2019. Climatic controls of decomposition drive the global biogeography of forest-tree symbioses. - Nature 569: 404-408.

Stoll, P. and Weiner, J. 2000. A neighborhood view of interactions among individual plants. - In: Dieckman, U., Law, R., Metz, J.A.J. (ed.) The geometry of ecological interactions: simplifying spatial complexity. Cambridge University Press, pp. 11-27.

Szabo, G. and Czaran, T. 2001. Defensive alliances in spatial models of cyclical population interactions. - Phys. Rev. E. 64: 1-4.

Taneyhill, D. E. 2000. Metapopulation dynamics of multiple species: The geometry of competition in a fragmented habitat. - Ecol. Monogr. 70: 495-516.

Tilman, D. 1982. Resource competition and community structure. - Prinecton Univ. Press. Tilman, D. 2004. Niche tradeoffs, neutrality, and community structure: A stochastic theory of resource competition, invasion, and community assembly. - P. Natl. Acad. Sci. USA 101: 10854-10861.

'This article is protected by copyright. All rights reserved.' 
Turchin, P. 2015. Quantitative analysis of movment: Measuring and modeling population redistribution in animals and plants. - Beresta Books.

Ulrich, W. et al. 2014. Matrix models for quantifying competitive intransitivity from species abundance data. - Oikos 123: 1057-1070.

Ulrich, W. et al. 2016. Species interactions and random dispersal rather than habitat filtering drive community assembly during early plant succession. - Oikos 125: 698707.

Ulrich, W. et al. 2017. Competitive interactions change the pattern of species cooccurrences under neutral dispersal. - Oikos 126: 91-100.

Vandermeer, J. H. 1969. The Competitive Structure of Communities: An Experimental Approach with Protozoa. - Ecology 50: 362-371.

Vandermeer, J. 2011. Intransitive loops in ecosystem models: From stable foci to heteroclinic cycles. - Ecol. Complex 8: 92-97.

Wang, X. G. et al. 2010. Spatial distributions of species in an old-growth temperate forest, northeastern China. - Can. J. Forest. Res. 40: 1011-1019.

Webb, S. D. et al. 2007. Host-parasite interactions between the local and the mean-field: How and when does spatial population structure matter? - J. Theor. Biol. 249: 140152.

Yang, Y. H. et al. 2014. Can we use disease to control biological invasion? - A theoretical research. - Ecol. Model. 277: 97-107.

Yang, Y. H. et al. 2019. Spatiotemporal landscape disturbance contributes to the suppression of competing invaders. - Ecol. Model. 393: 76-84. 
Ying, Z. X. et al. 2014. Species coexistence in a lattice-structured habitat: Effects of species dispersal and interactions. - J. Theor. Biol. 359: 184-191.

Yitbarek, S. and Vandermeer, J. H. 2017. Reduction of species coexistence through mixing in a spatial competition model. - Theor. Ecol. 10: 443-450.

Zhang, F. et al. 2006. Spatiotemporal dynamics and distribution patterns of cyclic competition in metapopulation. - Ecol. Model 193: 721-735. 


\section{Online supplementary materials:}

Appendix S1: Building a 3-species system with pair approximation.

Appendix S2: Calculation of the change in invasion growth rate under pair approximation.

Appendix S3: Calculation of the change in invasion growth rate under mean-field assumption.

Figure S1: The sensitivity of the change in invasion growth rate to changes in the intransitivity $\left(\theta_{3}\right)$ and niche overlap $(\mathrm{k})$ after the removal of one species. 


\section{Figure Legends}

Figure 1: A schematic illustration of local interactions in our model formulated using pair approximation. The cell dynamics include three processes: birth, intraspecific and interspecific competition. Symbols $i, j, l$ (and 0) represent cell states. Arrows with the corresponding probabilities indicate the state transition of the central cell from birth and mortality from intra- and interspecific competition. The central cell and the white cell form a pair in the pair approximation. Grey cells are other neighbouring cells to the central cell but are not part of the focal pair. Note, $\lambda=1 / z$, where $z$ is the number of neighbouring cells (we chose the von Neumann neighbourhood, $z=4$ ).

1) Birth

2) Intraspecific
competition

3) Interspecific competition
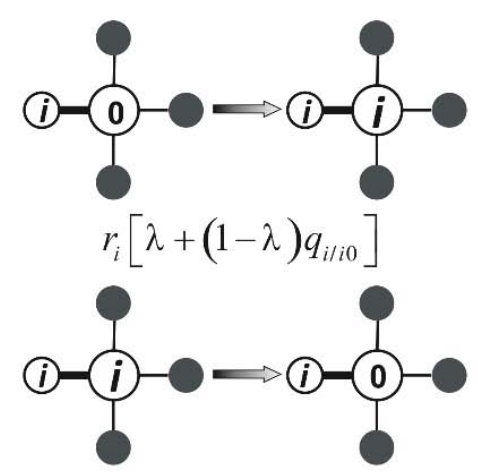

$$
\alpha_{i i}\left[\lambda+(1-\lambda) q_{i / i i}\right]
$$

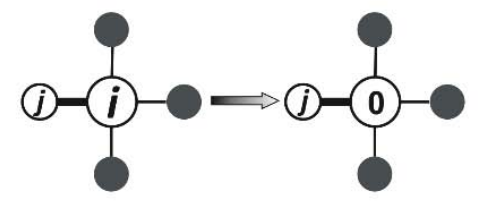

$\alpha_{i j}\left[\lambda+(1-\lambda) q_{j / j i}\right]$

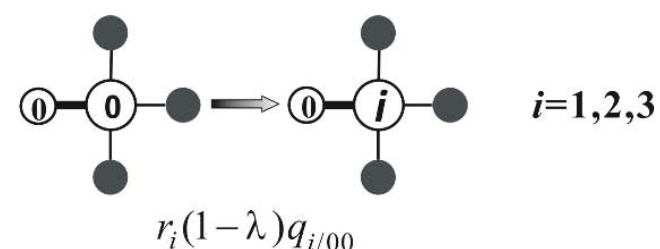

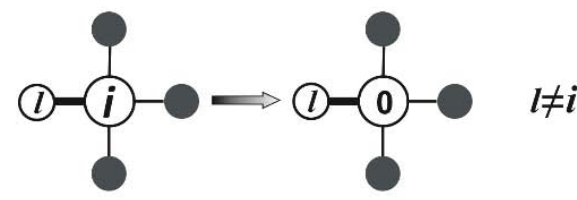

$\alpha_{i i}(1-\lambda) q_{i / l i}$

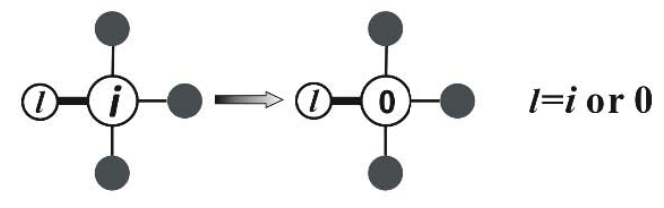

$\alpha_{j i}(1-\lambda) q_{j / l i}$

'This article is protected by copyright. All rights reserved.' 
Figure 2: The spectrum of transitive-intransitive continuum in a three-species system. Arrows represent the competition between two species and point at the weaker competitor, with the formulae for net competition strength provided. Double lines represent negligible or equal net competition. From left to right, intransitivity $\left(1-\theta_{3}\right)$ increases from $-(1-\theta) / 2$ to $(1-\theta)$, shifting a transitive network to an intransitive loop.
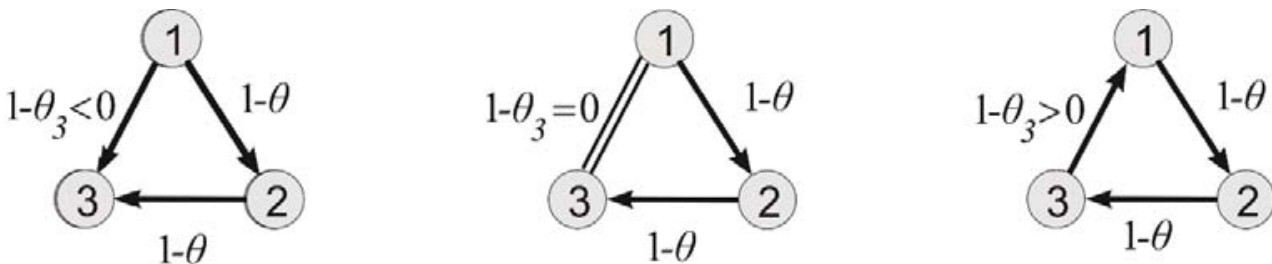

Transitive

Weak Intransitive

Intransitive 
Figure 3: The influence domain of competitive intransitivity on species coexistence. a) A heat map of metric $\overline{\Delta R}$ as a function of transitivity and niche overlap. Colour bar: blue colours represent $\overline{\Delta R} \leq 0$ while reddish colours represent $\overline{\Delta R}>0$. b) Parameter range for coexistence, derived by solving the parameter region for $R_{i}>0 \quad(i=1,2,3)$. The entire parameter space was divided into two areas: coexistence and extinction. Transitivity ranged within $[\theta, 3 / 2-\theta / 2]$, with $\theta=0.6, r_{1}=r_{2}=r_{3}=0.5, m_{1}=m_{2}=$ $m_{3}=0.01$.
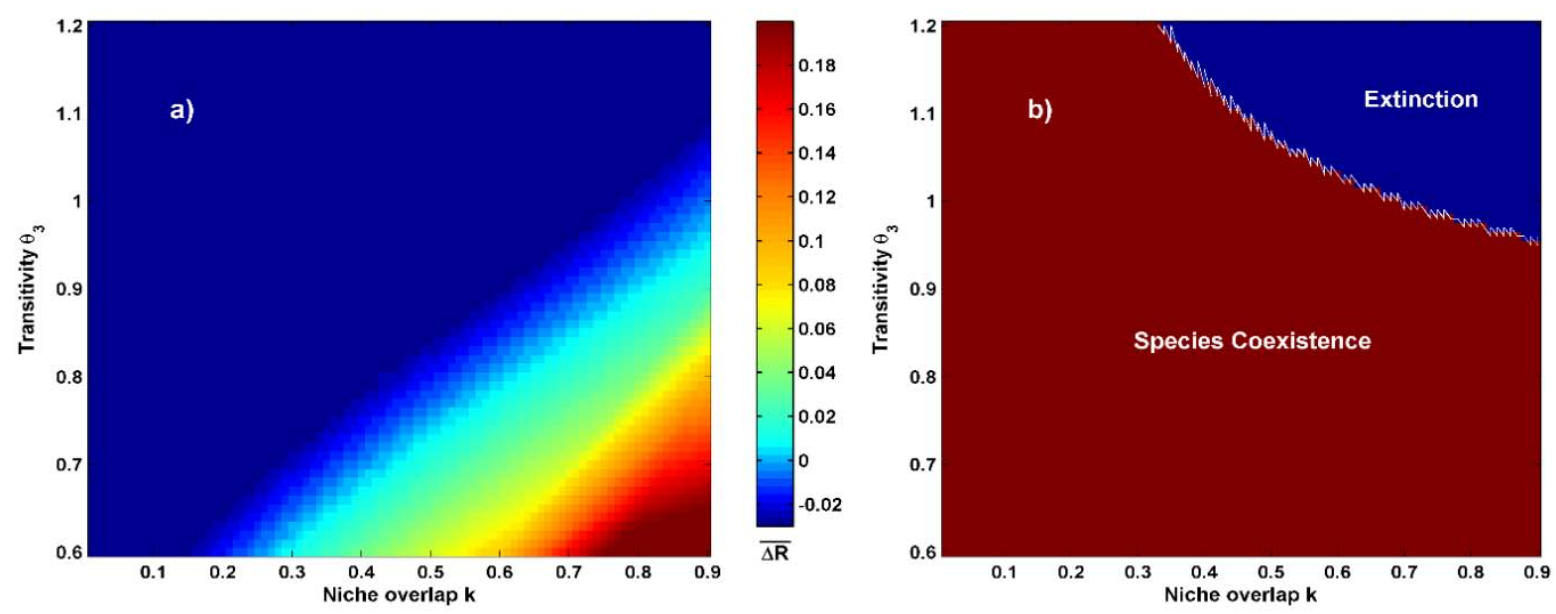

'This article is protected by copyright. All rights reserved.' 
Figure 4: The influence domain of competitive intransitivity at different levels of competitive symmetry $(\theta=0.3,0.5,0.6,0.7)$ and model assumptions (PA: pair approximation versus MFA: mean-field assumption). White lines separate coexistence from extinction domains. Other parameter values are the same as in Fig.3.
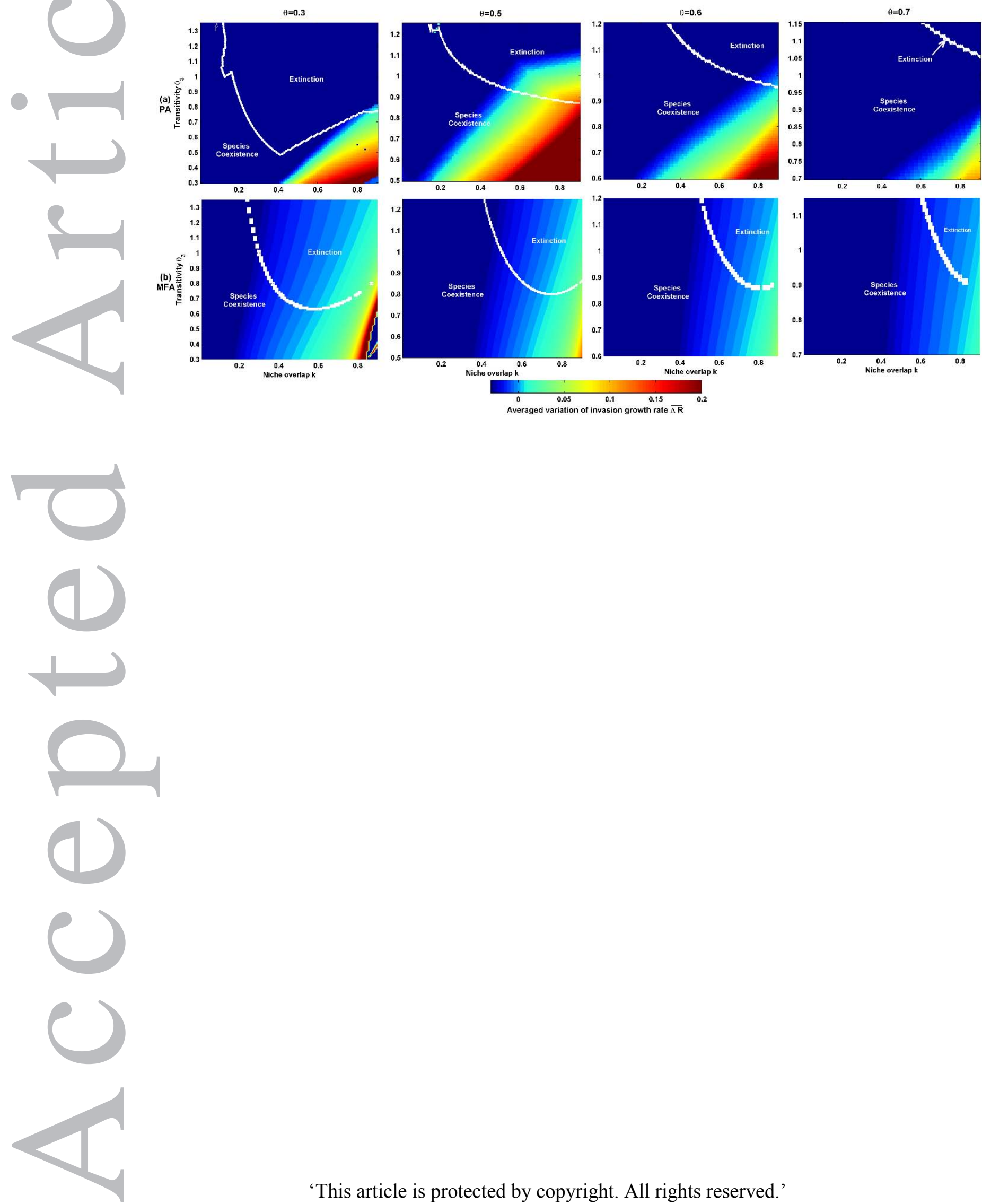

'This article is protected by copyright. All rights reserved.' 
Figure 5: Spatial patterns at equilibrium against niche overlap $k$. (a)The ratio of intraover interspecific interactions, $q_{i / i}^{*} /\left(q_{j / i}^{*}+q_{l / i}^{*}\right)$; (b) The probability of neighbouring empty cells, $q_{0 / i}^{*}$. Lines with different colours correspond to different competitive symmetries $(\theta)$. We chose $\theta_{3}=\theta$ for a pure intransitive loop. Other parameter values are the same as in Fig.3.
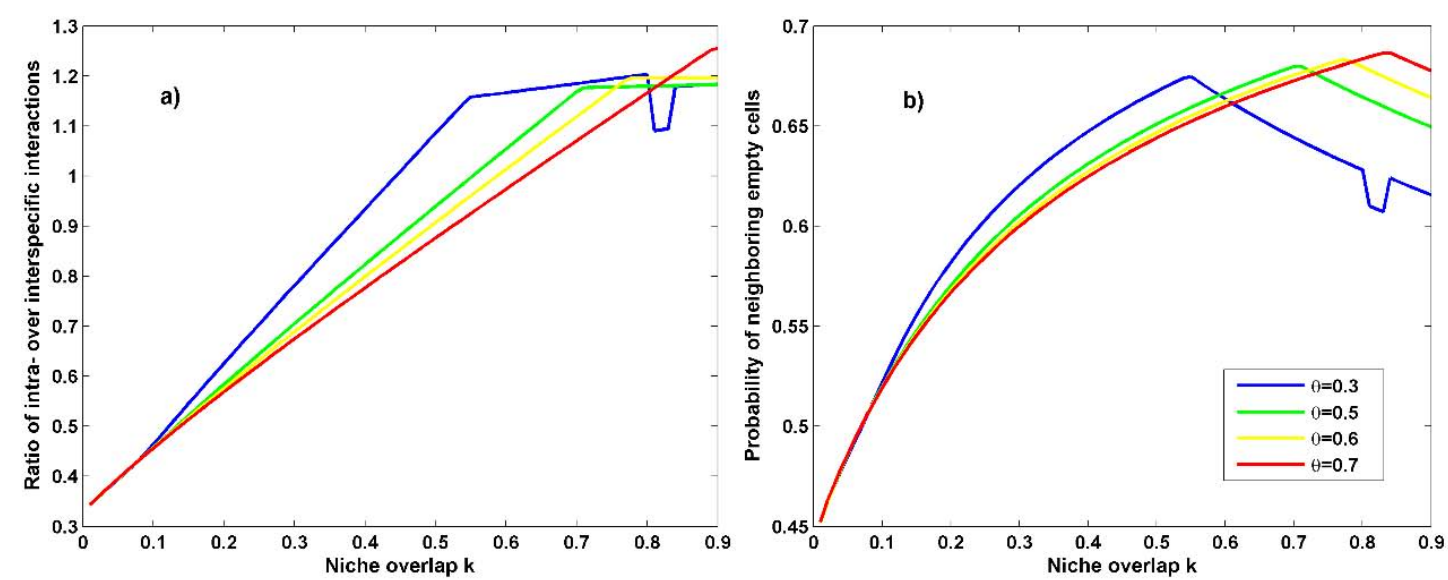

'This article is protected by copyright. All rights reserved.' 
Figure 6: Spatial dynamics of the cellular automata under three levels of niche overlap $(k=0.01,0.25,0.8)$. Top four rows exhibit snapshots of the spatial distributions at four time steps: $t=1,10,100,15000$. Dark blue: 0 (empty cells); light blue: species 1; yellow: species 2; red: species 3 . The last row presents corresponding plots of population dynamics. Competitive transitivity $\theta_{3}=\theta=0.6$. The dimension of the lattices is $100 \times 100$. Other parameter values are the same as in Fig.3.

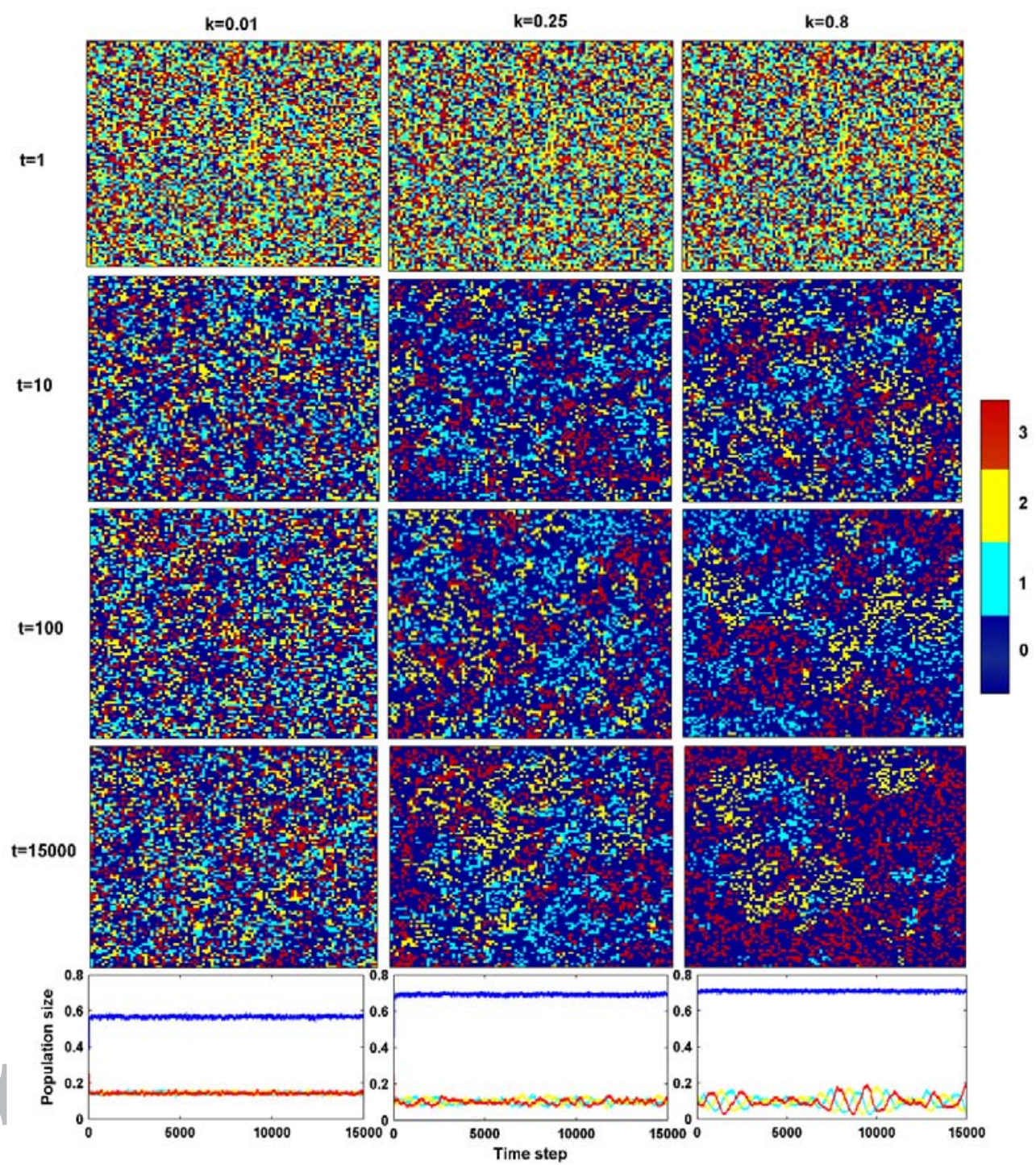

'This article is protected by copyright. All rights reserved.' 
Table Legemd

Table 1: Updating probabilities of the cellular automation from the states along the rows to the states along the columns.

\begin{tabular}{l|c|c|c|c} 
& \multicolumn{1}{c}{ Empty (0) } & Species 1 & Species 2 & Species 3 \\
\hline $\begin{array}{l}\text { Empty } \\
\text { (0) }\end{array}$ & $1-r_{1} \frac{\sum s p_{1}}{Z}-r_{2} \frac{\sum s p_{2}}{Z}-r_{3} \frac{\sum s p_{3}}{Z}$ & $r_{1} \frac{\sum s p_{1}}{Z}$ & $r_{2} \frac{\sum s p_{2}}{Z}$ & $r_{3} \frac{\sum s p_{3}}{Z}$ \\
\hline Species & $\Delta_{1}:=m_{1}+\alpha_{11} \frac{\sum s p_{1}}{Z}+\alpha_{21} \frac{\sum s p_{2}}{Z}+\alpha_{31} \frac{\sum s p_{3}}{Z}$ & $1-\Delta_{1}$ & - & - \\
\hline Species 2 & $\Lambda_{2}:=m_{2}+\alpha_{12} \frac{\sum s p_{1}}{Z}+\alpha_{22} \frac{\sum s p_{2}}{Z}+\alpha_{32} \frac{\sum s p_{3}}{Z}$ & - & - \\
\hline Species & $1_{3}:=m_{3}+\alpha_{13} \frac{\sum s p_{1}}{Z}+\alpha_{23} \frac{\sum s p_{2}}{Z}+\alpha_{33} \frac{\sum s p_{3}}{Z}$ & - & $1-\Delta_{2}$ & - \\
\hline
\end{tabular}

Note: $\sum s p_{i}\left(i=\quad J\right.$ stands for the total number of species $i$ among the nearest $z$ number of neighbours. In addition, we choose $r_{1}=r_{2}=r_{3}=$ $r$ and $m_{1}=m=m_{3}=m$.

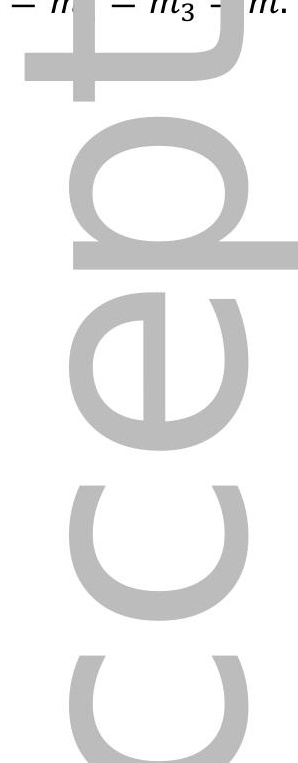

